\title{
Magnetic properties and giant magnetoimpedance effect for CoFeMoSiB surface modified amorphous ribbons covered by water based ferrofluid
}

\author{
Zahra Lotfollahi I,*, Ahmad Amirabadizadeh ${ }^{1}$, Alexander P. Safronov ${ }^{2,3}$, Galina V. Kurlyandskaya ${ }^{2,4}$ \\ ${ }^{1}$ University of Birjand, 97175-615, Birjand, Iran \\ ${ }^{2}$ Ural Federal University, Mira str., 19, Ekaterinburg, 620002, Russia \\ ${ }^{3}$ Institute of Electrophysics, Amundsen str., 106, Ekaterinburg, 620016, Russia \\ ${ }^{4}$ Universidad del País Vasco UPV-EHU, Depto Electricidad y Electrónica, Leioa, 48940, Spain
}

\begin{abstract}
Giant magnetoimpedance (GMI) effect is a powerful technique for magnetic label detection. Cobased amorphous ribbons are cheap materials showing high GMI effect at low operation frequencies for close to zero magnitostriction compositions. In this work magnetic properties and GMI were studied for $\mathrm{CoFeMoSiB}$ amorphous ribbons in as-quenched and surface modified states without and in the presence of water-based ferrofluid with electrostatic stabilization of $\gamma-\mathrm{Fe}_{2} \mathrm{O}_{3}$ nanoparticles. Surface modification by ultrasound treatment resulted in appearance of round defects with average diameter of about 150 micrometers. The GMI difference for as-quenched ribbons in absence and in the presence of ferrofluid was measured for the frequency range of 0.5 to $10 \mathrm{MHz}$. Although proposed surface modification by the ultrasound treatment did not improve the sensitivity limit for ferrofluid detection, it did not decrease it either. Observed changes of GMI are useful for understanding of functionality of GMI biosensors.
\end{abstract}

\section{Introduction}

Giant magnetoimpedance (GMI) effect in soft ferromagnets is promising for applications in the area of small magnetic field sensors including magnetic biosensors [1-4]. Magnetoimpedance phenomenon is a change of the total impedance of ferromagnetic conductor under application of an external magnetic field when high frequency alternating current flows through it [2]. Different kinds of materials were proposed for GMI biosensors [3, 5-7]. The idea of GMI biosensor based on amorphous ribbon is attractive because this kind of material is cheap and sensitive element can be designed as a disposable strip [5]. Two types of GMI-based biosensors are discussed in a literature. The first one is related to magnetic label detection [3] and the second is for label free detection process [8]. In the second case the rapidly quenched ribbon sensitive element slightly changes the mass during the sensing process. The surface layer properties of the rapidly quenched ribbon differ from the properties of the central part. Therefore, gradual removal of the surface layer causes the change of the effective magnetic anisotropy and GMI [8].

Such a parameter as surface roughness is important in the case of amorphous ribbon based biosensors. On one hand, it might be seen as an obstacle in the case of magnetic label detection influencing the sensitive element response (due to strong contribution of the surface anisotropy). On the other hand, one can expect

\footnotetext{
Corresponding author: 1otfollahi@gmail.com
}

the improvement of the magnetic flux closure for the rough surface with magnetic ferrofluid spread on it. The origin of sensitivity is still not fully understood and it depends on surface features, size and agglomeration state of magnetic labels and other parameters $[6,9]$.

Mechanical processing of the surface modification of the amorphous materials is practically impossible due to their physical properties. For the magnetic detection of the MNPs they usually select $\mathrm{Mo}$ or $\mathrm{Cr}$ doped compositions with enhanced corrosion stability. Consequently, chemical processing of these kinds of ribbons (like lithographic process including chemical etching) is also not simple. There were attempts to use advanced technique of lithography for surface defects creation or even mechanical drilling $[10,11,12]$ but employment of such an expensive technique makes whole device less adapted for applications. Search for new methods of creation of artificial surface defects for the ribbons with enhanced corrosion stability is therefore a challenge.

In this work, spherical magnetic nanoparticles were fabricated by the laser target evaporation. Electrostatically stabilized water-based suspensions were prepared on the basis of obtained MNPs. Their physical properties were studied by different techniques prior to GMI measurements without and with immersing the sensitive element into a magnetic suspension. CoFeMoSiB amorphous ribbon GMI sensitive element was either in as-quenched or in ultrasound treatment surface modified state. 


\section{Experimental}

Iron oxide nanoparticles (MNPs) were synthesized by the laser target evaporation (LTE) method using Ytterbium $(\mathrm{Yb})$ fiber laser with $1.07 \mu \mathrm{m}$ wavelength. More details of LTE technique can be found elsewhere [12-13]. Structural studies of as prepared MNPs were performed by transmission electron microscopy (TEM) using a JEOL JEM2100 microscope operating at $200 \mathrm{kV}$. The X-ray diffraction (XRD) studies of the MNPs and the ribbons were performed by DISCOVER D8 (Bruker) diffractometer using $\mathrm{Cu}-\mathrm{K} \alpha$ radiation (wave lengths $\lambda=1.5418 \AA$ ). Electrostatically stabilized ferrofluid (FF) was prepared by ultrasound treatment using sodium citrate solutions $(5 \mathrm{mM})$ in distilled water. The final concentration of MNPs in ferrofluid was $5.0 \%$. The specific surface area of MNPs was measured by the lowtemperature sorption of nitrogen (Brunauer-EmmettTeller physical adsorption method, BET) using Micromeritics TriStar 3000 analyzer.

$\mathrm{Co}_{68.6} \mathrm{Fe}_{3.9} \mathrm{Mo}_{3.0} \mathrm{Si}_{12.0} \mathrm{~B}_{12.5}$ amorphous $0.7 \mathrm{~mm}$ wide and $20 \mu \mathrm{m}$ thick ribbons were prepared by rapid quenching onto $\mathrm{Cu}$ weal technique (tangential velocity of $\sim 30 \mathrm{~m} / \mathrm{s}$ ). Saturation magnetostriction of this ribbon was close to zero [14]. Surface modification of the amorphous ribbon was done in an ultrasonic bath with $5 \% \quad \mathrm{H}_{3} \mathrm{PO}_{4}$ acid concentration during 120 minutes treatment for creation of equidistantly distributed artificial surface defects. As-quenched ribbon was called as AQ, surface modified as SM.

Surface features of the ribbons were studied by the scanning electron microscopy (SEM) using by TM3000 HITACHI instrument. Magnetic measurements at room temperature were done by vibrating sample magnetometer (VSM, Lake Shore 7404). The total impedance $(Z)$ was measured by the four-point technique for different frequencies of 0.5 to $10 \mathrm{MHz}$ and of the driving current peak-to-peak intensity of $5 \mathrm{~mA}$. Giant magnetoimpedance ratio for total impedance was defined as follows: $\Delta \mathrm{Z} / \mathrm{Z}=100 \times\left(\mathrm{Z}(\mathrm{H})-\mathrm{Z}\left(\mathrm{H}_{\max }\right)\right) / \mathrm{Z}\left(\mathrm{H}_{\max }\right)$, where $\mathrm{H}_{\max }=200$ Oe. For GMI measurements in presence of water-based ferrofluid with MNPs of the ferrofluid 3.5 $\mathrm{cm}$ long sample was placed into a plastic tube of $30 \mathrm{~mm}$ length and $1 \mathrm{~mm}$ in diameter. Ribbon was located in centre of a tube filled with ferrofluid.

\section{Results and discussion}

Fig. 1 shows XRD spectrum with evidence of amorphous structure of the ribbon (very wide peak near $45 \pm 3^{\circ}$ for $2 \theta$ angles). The SEM micrographs (Fig. 1, insets) show the surface morphology before and after ultrasound treatment in acid revealing sufficiently smooth surface of the bright side of AQ ribbon with typical for rapid quenching technique defects in the direction of the ribbon displacement during the solidification. SM ribbon has surface defects which can be described as round indentations with average diameter of about 150 micrometers with no anisotropy in the shape of the defects or their orientation. Surface defects were equidistantly separated from each other as a
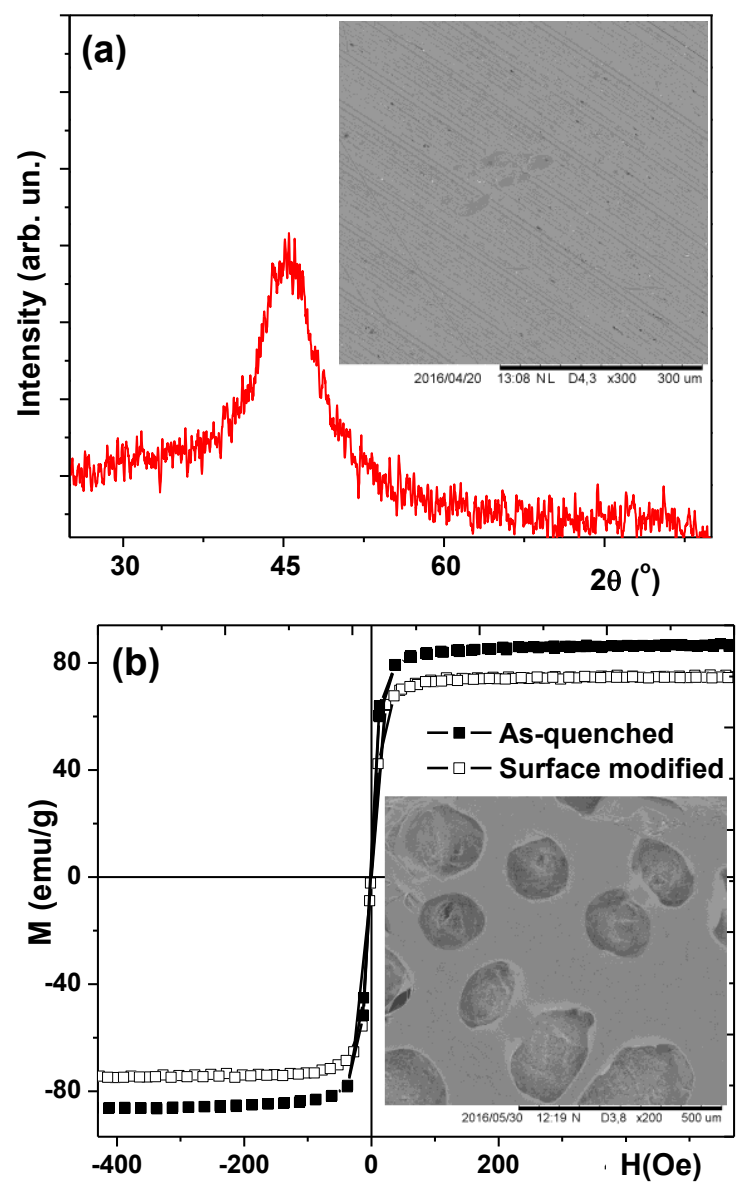

Fig. 1. XRD pattern for $\mathrm{Co}_{68.6} \mathrm{Fe}_{3.9} \mathrm{Mo}_{3.0} \mathrm{Si}_{12.0} \mathrm{~B}_{12.5}$ amorphous ribbon in as-quenched state. Inset shows SEM image of bright side of the AQ ribbon (a). VSM hysteresis loops for AQ and SM states, inset shows SEM image of bright side of the SM ribbon (b).

result of self assembling process and approximately $50 \%$ of the surface was covered by the defects. The surface layer of about 2-3 $\mu \mathrm{m}$ was most affected part of the ribbon in the course of the ultrasound treatment. Magnetic characterization for AQ and SM ribbons shows in both cases quite rapid saturation approach and round shoulder shape in the saturation approach indicating mixed anisotropy with domination of the longitudinal one. Part of the contribution (transverse anisotropy component) can come from the surface anisotropy.

Surface modification by the controlled ultrasound treatment results in a slight decrease of saturation magnetization from 87 to $76 \mathrm{emu} / \mathrm{g}$ and does not affect the coercivity. Saturation magnetization decrease is consistent with surface modification which includes the removal of the initial surface layer and formation of a passivation layer with lower saturation magnetization, comparing with the material of the AQ ribbon.

XRD spectra of MNPs gave a mean crystallite size for air-dried LTE MNPs of $19 \pm 2 \mathrm{~nm}$ (log-normal distribution) in a good agreement with TEM data and specific surface area evaluation (Fig. 2). Although the experimental XRD data were well fitted by the magnetite database, it was impossible to distinguish the magnetite and maghemite on the basis of XRD studies solely. The 
chemical composition of LTE MNPs was determined by the combination of redox titration and the lattice period analysis provided by XRD: it was close to the stoichiometric maghemite $\left(\mathrm{Fe}_{2.72} \mathrm{O}_{4}\right)$. Also we can see that the $\mathrm{M}_{\mathrm{s}}$ of the MNPs is about $57 \mathrm{emu} / \mathrm{g}$. This value is lower than for the bulk maghemite as to expect for the MNPs of the observed average size due to nanoscaling effects [12]. At the same time magnetic measurements confirm well the concentration of the MNPs of ferrofluid previously defined by chemical titration technique.

Fig 3. shows frequency dependences of the maximum GMI ratio $(\Delta Z / Z)_{\max }$ for $A Q$ and $S M$ ribbons in the presence and absence of FF: until $\mathrm{f}=5 \mathrm{MHz}$, GMI maximum increases and afterwards it changes very little.
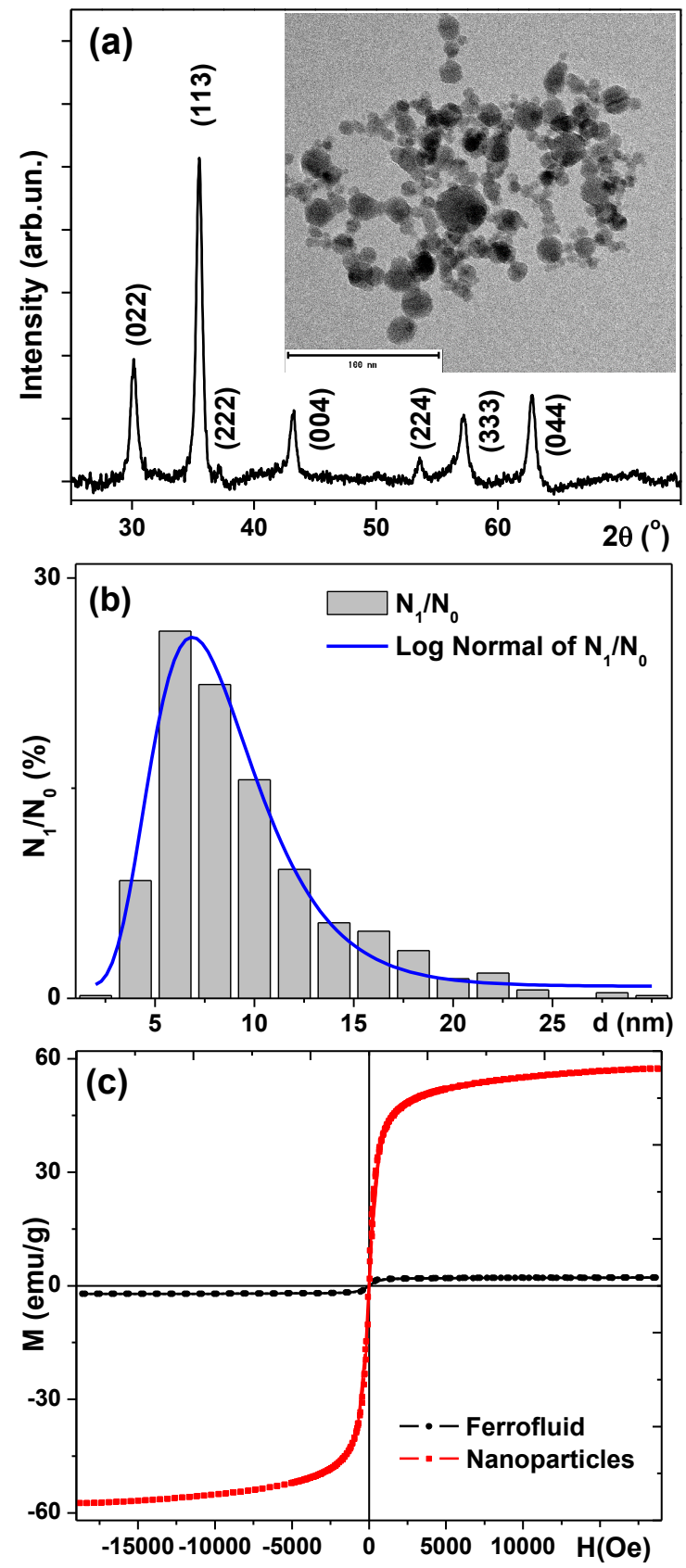

Fig. 2. XRD pattern for LTE MNPs from ferrofluid, inset shows TEM image of LTE MNPs (a). Histogram of particle size distribution and log-normal fits (blue line) (b). Hysteresis loops of as-prepared LTE MNPs and electrostatically stabilized water-based suspension of LTE MNPs (c).
This behavior is consistent with skin depth changes: the condition of strong skin effect appears for the frequencies above 4-5 MHz. In a frequency range 0.5-10 $\mathrm{MHz}$ GMI responses of the as-quenched and surface modified ribbons had very similar shapes but the GMI values were slightly higher for $A Q$ ribbons. $M(H)$ hysteresis loops are similar. We therefore assign GMI difference to the surface anisotropy contribution and dynamic magnetic permeability. An increase of the surface roughness and the partial removal of very thin surface layer during ultrasound treatment due to formation of the round defects can be a reason of $(\Delta \mathrm{Z} / \mathrm{Z})_{\max }$ value decrease. $(\Delta \mathrm{Z} / \mathrm{Z})_{\max }(\mathrm{f})$ curves were very
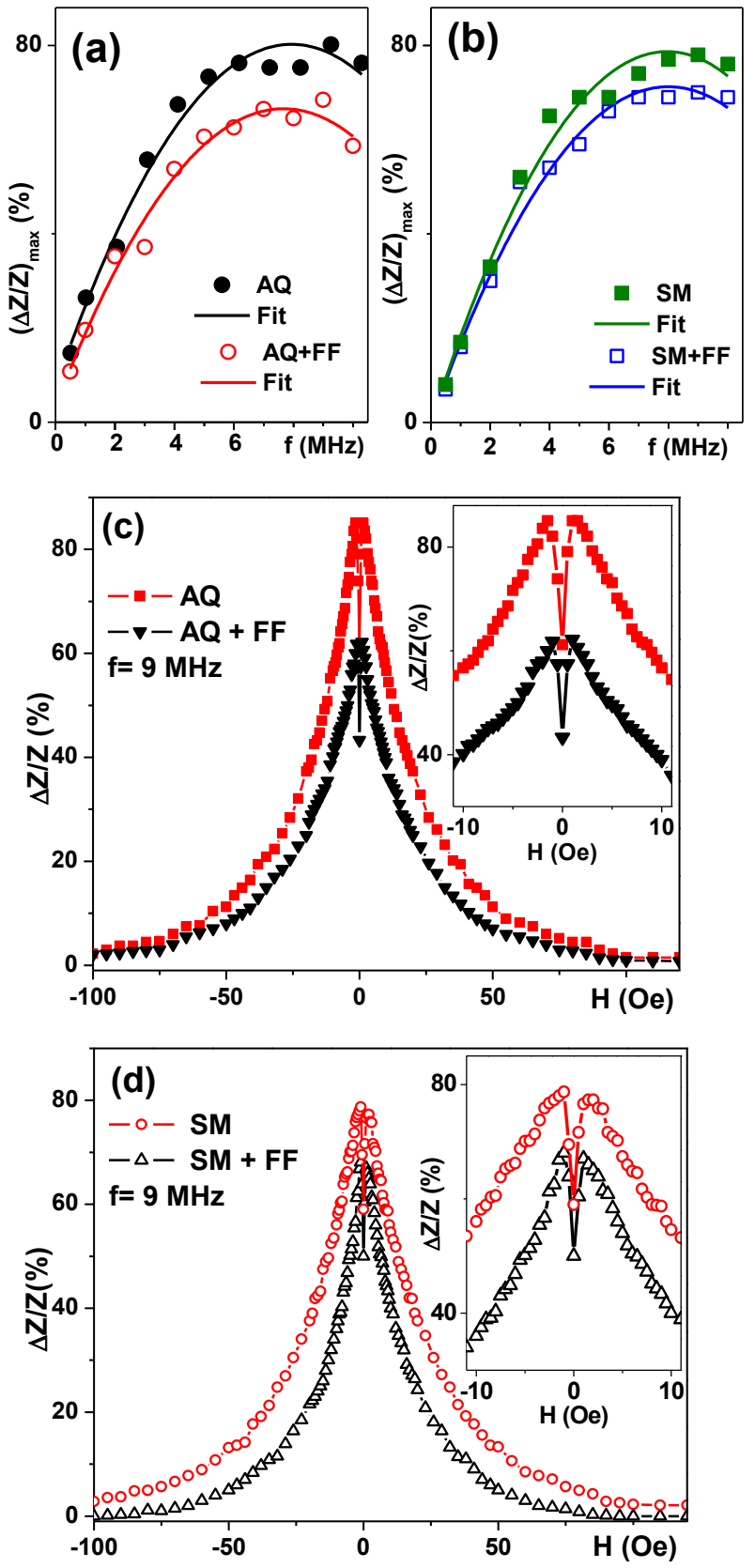

Fig. 3. Frequency dependences of the $(\Delta \mathrm{Z} / \mathrm{Z})_{\max }$ ratio in the presence and in absence of ferrofluid for as-quenched (a) and surface modified (b) and Field dependences of GMI ratio in the presence and in absence of ferrofluid for as-quenched (c) and surface modified (d) CoFeMoSiB amorphous ribbons. 
much affected by the presence of FF in AQ and lesser in $\mathrm{SM}$ ribbons. For AQ ribbons covering by the FF resulted in a decrease of GMI ratio in comparison with response without FF. The change in the GMI ratio due to the presence of the MNPs can be explained by the effect of their fringe fields on the superposition of the applied direct current magnetic field and the induced transverse alternating current field created by the driving current.

Field dependences of GMI ratios (Fig.3) shows that the general shapes of the $\Delta \mathrm{Z} / \mathrm{Z}(\mathrm{H})$ in both cases (AQ and $\mathrm{SM}$ ribbons) correspond well to the longitudinal effective magnetic anisotropy (one-peak GMI curve) [13-14]. At the same time the appearance of double peak shape related to small contribution of the surface component [8] of transverse magnetic anisotropy is evident in a small field. Increase of the frequency from 3 to $9 \mathrm{MHz}$ results in the change of the shape of $\Delta \mathrm{Z} / \mathrm{Z}(\mathrm{H})$ curves and double peak behaviour become more obvious: dip of the double-peak at zero field increases with the frequency increase. For the frequency of $3 \mathrm{MHz}$ maximum GMI in presence of FF decreases from $65 \%$ to $46 \%$ and for surface modified ribbon decreases from $53 \%$ to $43 \%$, i.e. GMI response of AQ ribbon offers more stable detection opportunities with better noise-tosignal characteristics. For frequency $9 \mathrm{MHz}$ similar conclusion can be made as the maximum GMI in presence of FF for AQ ribbon decreases from $85 \%$ to $61 \%$ and for SM ribbon decreases from $78 \%$ to $68 \%$.

Creation of round defects makes ribbon less sensitive to the presence of FF but the origin of this sensitivity is not trivial. The GMI sensing process counts with the magnetic field of the alternating current passing through the ribbon, the constant external magnetic field applied during GMI measurements for the change of magnetic permeability of the sensitive element, the stray fields created by MNPs and stray fields of round created defects. Surface modification changes demagnetizing fields: defects corresponding to rapid solidification become removed but new defects in the shape of round indentations are formed. The indentations are large, comparing with typical quenching defects but out of the border of the round defect the surface roughness become even smaller. We can suppose that the flux closing by the magnetic chains formed in FF [15] contributes to GMI.

\section{Conclusions}

Amorphous $\mathrm{Co}_{68.6} \mathrm{Fe}_{3.9} \mathrm{Mo}_{3.0} \mathrm{Si}_{12.0} \mathrm{~B}_{12.5}$ ribbons with good corrosion stability were prepared by rapid quenching technique. Magnetic and GMI properties were studied in as-prepared state and after creation of artificial surface defects by the ultrasound treatment in the acid.

GMI responses in as-quenched and surface modified state in the absence and in presence of LTE MNPs based ferrofluid model the magnetic biosensor functionality for magnetic labels detection. GMI curves were affected by the presence of ferrofluid in as-quenched and much less in the surface modified ribbon cases. Covering by the ferrofluid resulted in a decrease of GMI ratio in all frequency range. For the frequency $3 \mathrm{MHz}$ maximum
GMI in presence of FF decreases from $65 \%$ to $46 \%$ and for surface modified ribbon decreases from $53 \%$ to $43 \%$. GMI response of AQ ribbon shows better noise-to-signal characteristics. The change in the GMI ratio with ferrofluid covering can be explained by the effect of fringe fields of MNPs and magnetic flux closure due to the MNPs chains formation. Obtained results can be useful for the development of the magnetic biosensor prototype with cheap disposal amorphous ribbon based GMI sensitive element operating at low frequencies of the order of $5 \mathrm{MHz}$.

The work was supported in part by the Government of the BC is acknowledged for financial support under the Elkartek Program, the Project Micro4Fab (KK-2016/00030) and the Ministry of Education and Science of the RF, Project $\mathrm{N}^{\circ}$ 055, within the state job 3.6121.2017/8.9. We thank I.V. Beketov, A.I. Medvedev and A. Larranga for special support. Selected measurements were made at SGIKER services of UPV/EHU.

\section{References}

1. R.S. Beach and Berkowitz, A.E., Appl. Phys. Lett. 64, 3652-3654 (1994).

2. L.V. Panina and K. Mohri, Sens. Act. A 81, 71-77 (2000).

3. G.V. Kurlyandskaya and V.I. Levit, Mater. Sci. Eng. C 27 495-503 (2007).

4 A. Antonov, S. Gadetsky, A. Granovsky, A. Diachkov,M. Sedova, N. Perov, T. Furmanova, A.Lagarkov, Physica A 241, 414 (1997).

5. G.V. Kurlyandskaya, E. Fernandez, A.P. Safronov, A.V. Svalov, I.V. Beketov, A. Burgoa Beitia, A. Garcia-Arribas, F.A. Blyakhman, Appl. Phys. Lett. 106, 193702 (2015).

6. F. Blanc-Beguin, S. Nabily, J. Gieraltowski, A. Turzo, S. Querellou, P.Y. Salaun, J. Magn. Magn. Mater. 321, 192-197 (2009).

7. H. Chiriac, M. Tibu, A.E. Moga, and D. D. Herea,

J. Magn. Magn. Mater. 293, 671 (2005).

8. G.V. Kurlyandskaya, V. Fal Miyar, V., A. Saad, E. Asua, and J. Rodriguez, J. Appl. Phys. 101(5), 054505 (2007).

9. J. Devkota, A. Ruiz, P. Mukherjee, H. Srikanth, and M. H. Phan, IEEE Trans. Magn. 49, 4060 (2013).

10. S. Kumar Manna and V. Srinivas J. Magn. Magn. Mater. 418, 62-67 (2016).

11. A. Garcia-Arribas, M. Goiriena-Goikoetxea, E. Fernández, and J.M. Barandiarán IEEE Trans. Magn. 53, 400310 (2017).

12. J.P. Novoselova, A.P. Safronov, O. Samatov, I.V. Beketov, H. Khurshid, Z. Nemati, H. Srikanth, T. P. Denisova, R. Andrade, and G.V. Kurlyandskaya, IEEE Trans. Magn. 50, 4600504 (2014).

13. L. Kraus, Sens. Act. A 106, 187-194 (2003).

14. Z. Lotfollahi, A. García-Arribas, A. Amirabadizadeh, I. Orue, and G.V. Kurlyandskaya, J. Alloys and Compounds, 693, 767-776 (2017).

15. A. Hubert and R. Schäfer, Magnetic Domains. Berlin, Germany: Springer (1998). 